\title{
Further Studies of the Photometric Variability of Bright Pulsating Red Giants
}

\author{
John R. Percy, M. Mashintsova, C. O. Nasui, R. Palaniappan, and R. Seneviratne \\ Department of Astronomy and Astrophysics, University of Toronto, Toronto ON Canada M5S 3H4; john.percy@utoronto.ca \\ AND \\ GREGORY W. HENRY \\ Center of Excellence in Information Systems, Tennessee State University, Nashville TN 37209 USA; henry@ schwab.tsuniv.edu \\ Received 2007 April 13; accepted 2008 March 27; published 2008 April 22
}

\begin{abstract}
Long-term photoelectric $V$ photometry of 13 pulsating red giants (TV Psc, EG And, RZ Ari, $\eta$ Gem, V614 Mon, RS Cnc, VY UMa, FS Com, SW Vir, R Lyr, EU Del, V1070 Cyg, and W Cyg) from a robotic telescope (APT) and from the photoelectric photometry program of the American Association of Variable Star Observers has been merged to form data sets that are significantly longer and denser than the subsets, and have been analyzed using Fourier and self-correlation techniques. Improved values of the pulsation periods, and especially of the enigmatic long secondary periods (LSPs), have been determined. Most of the stars also show very slow, small variations in amplitude and mean magnitude on timescales of thousands of days. The amplitude variability has also been studied, using both least-squares and wavelet analysis; the dominant amplitude variability is on a timescale of years. The period of EU Del shows neither long-term secular changes nor random cycle-tocycle fluctuations. APT VRI photometry for 16 stars has been analyzed: for both the pulsation periods and for the long secondary periods (with one notable exception-EG And), the amplitude decreases with increasing wavelength.
\end{abstract}

Online material: color figures

\section{INTRODUCTION}

Red giants cooler than spectral type K5III are variable in brightness. (Red giants warmer than K5III are microvariable, for different reasons; we shall not discuss them further here.) Pulsating red giants (PRGs) make up about $10 \%$ of the stars in the Bright Star Catalog (Hoffleit \& Jaschek 1991). They are stars with masses of up to a few solar masses, and temperatures of $4000 \mathrm{~K}$ and cooler, ascending the red giant or asymptotic giant branch in the H-R diagram. The $V$ amplitude of variability ranges from millimagnitudes at K5III to many magnitudes in the rarest, coolest, late-M-type Mira stars. The dominant periods range from 10 to over 200 days, and are consistent with low-order radial pulsation. Some PRGs pulsate in two or three different radial modes. About a third also have long secondary periods (hereafter LSPs), whose cause is unknown. The multiperiodicity and LSPs in larger-amplitude PRGs have been studied especially by Kiss et al. (1999). PRGs have been found, in large numbers, with all the properties mentioned above, in large-scale surveys such as the MACHO and OGLE microlensing surveys. See Percy et al. (2004), and other papers in that conference proceedings (Kurtz \& Pollard 2004), for specific references and brief reviews.

\section{PURPOSES OF THIS PROJECT}

We have previously analyzed the long-term variability of PRGs using photoelectric photometry from the American Association of Variable Star Observers (AAVSO) International Database (Percy et al. 1996, 2001a), and from an Automatic Photometric Telescope (APT) or robotic telescope (Percy et al. 2001b, hereafter PWH). The two data sets were analyzed independently. The AAVSO data have recently been reprocessed, subjected to additional quality controls, and made available online (Waagen 2006). For this reason, we thought it would be useful to merge the two data sets for the 13 stars in common, to see whether we could obtain additional or improved information, especially about their long-term and very long-term variability, from the longer denser data sets.

The data sets are complementary; for instance, the robotic telescope was not operational during the summer monsoon season in Arizona, whereas the AAVSO program was.

The lengths of our data sets are typically over 20 yearslonger than any other photoelectric data sets on bright PRGs. We have used the merged data sets to determine or redetermine various properties of the variability. We have also analyzed the APT $R I$ data to investigate the color-amplitude relation; previously, only the $V$ data had been analyzed. The study and 
TABLE 1

Periods (in Days) of 12 Small-Amplitude Pulsating Red Giants

\begin{tabular}{|c|c|c|c|}
\hline Star & Percy et al. (1996a, 2001a) & PWH & Present Result \\
\hline TV Psc & 53.11, 1400: & $55,570-1400$ & 55,550 \\
\hline EG And $\ldots \ldots \ldots$ & 29,240 & 29,242 & 29,244 \\
\hline RZ Ari $\ldots \ldots \ldots$ & 56:, 500: & 56.5 , shorter & $37.7,56.5,370$ \\
\hline$\eta$ Gem $\ldots \ldots \ldots$ & 239.8 & 234 , shorter? & 234, no LSP \\
\hline V614 Mon ..... & 80, long: & 80.7 & 90, 67?, no LSP \\
\hline RS Cnc $\ldots \ldots \ldots$ & 228, long: & 50-150:, 2000 & $225,137 ?$, no LSP \\
\hline VY UMa $\ldots . . .$. & $200::$ & 125, 190: & 125, 188:, no LSP \\
\hline FS Com $\ldots \ldots \ldots$ & 55.51, long: & 55.1, shorter, 600-750: & $38.2,55.4,680$ \\
\hline SW Vir $\ldots \ldots \ldots$ & 155 & 153.8 , long & 154, no LSP \\
\hline R Lyr & 53.41, long: & 46 and/or 64 & 46, 64.3, no LSP \\
\hline EU Del $\ldots \ldots \ldots$ & 62.74 & 62.3 , long & 61.6, no LSP \\
\hline V1070 Cyg ..... & 110,470 & $60,50 \pm$, complex & $64,100 ?, 42 ?$, no LSP \\
\hline W Cyg $\ldots . . . .$. & 120:, 260: & 130.4, complex & 131, 250:, no LSP \\
\hline
\end{tabular}

analysis of bright PRGs provide a model for analyzing the thousands of fainter PRGs that are being discovered in surveys, and for which the data sets may eventually be as long as ours.

\section{DATA}

The 13 stars in common to the two programs are listed in Table 1 and described below. The APT data are described by PWH; the data are publicly available. No changes have been made to the data since that time. PWH analyzed only the $V$ data; here, we analyze the $R$ and $I$ data also. The AAVSO data are described by Percy et al. (1996, 2001a). Since then, the data have been reprocessed, using better extinction and transformation coefficients, dealing with a few slightly variable comparison stars, and weeding out a few observations of less-than-optimal accuracy (Waagen 2006), and made publicly available on the AAVSO Web site. ${ }^{1}$ Unlike the APT photometry, the AAVSO photometry has continued, and so the merged data set is both larger (by up to 1.5 times as many points) and longer (by typically a factor of 1.8) than either of the individual data sets that we have previously analyzed.

The AAVSO photometry was differential $\Delta V$. It was reduced to $V$ (for comparison with visual observations of the same stars) by AAVSO staff, using the catalog magnitudes of the comparison stars. The APT photometry was also differential. We have reduced it to $V$ by adding the catalog magnitudes of the comparison stars. After merging the data for each star, we examined the merged data to ensure that there were no small offsets between the two data sets.

\section{ANALYSIS AND RESULTS}

\subsection{Short and Long Periods}

The data sets were subjected to Fourier analysis using the Period04 package from the University of Vienna (Lenz \&

\footnotetext{
${ }^{1}$ The AAVSO Web address is www.aavso.org.
}

Breger 2005) and to self-correlation analysis as described by Percy et al. (1996, 2001b). Coherent timescales produce minima in the self-correlation diagram (SCD) at multiples of the basic timescale. Their persistence reflects the degree of regularity of the variability. In this section, we describe the results for individual stars, and show a few representative power spectra and SCDs.

TV Psc.-The pulsation period is $55 \pm 1$ days, from both the power spectrum and the SCD. According to the SCD (Fig. 1), the LSP is 550 days, and this is one of three low-frequency peaks in the power spectrum, the others being 1 cycle/year aliases of this. PWH obtained periods of 55 and 570-1400 days.

$E G$ And.-The pulsation period is 29 days, and the long secondary period is 242 days. PWH obtained periods of 29 and 242 days. Because the binary period is $482.57 \pm 0.53$ days (Fekel et al. 2000), it appears that the LSP is associated with the binarity.

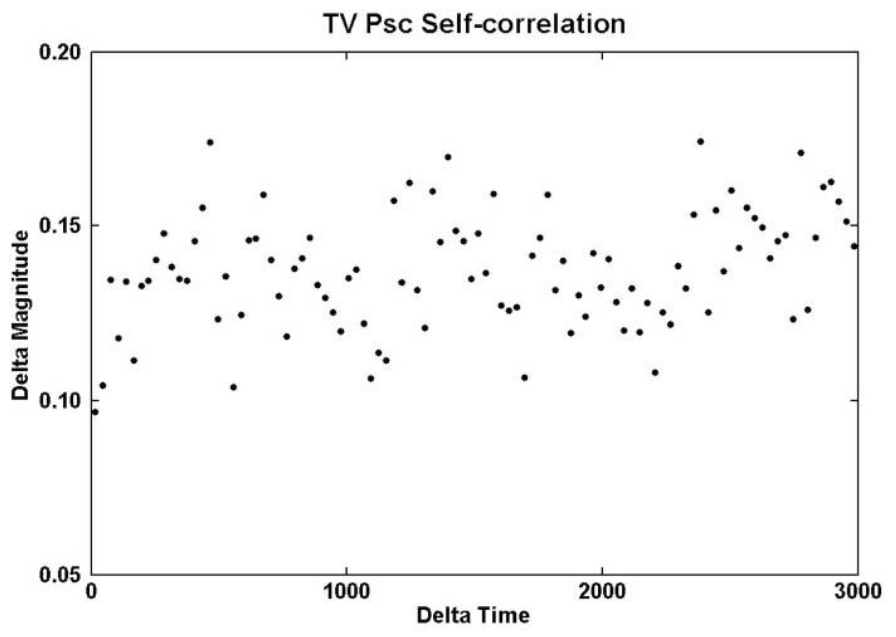

FIG. 1.-SCD of TV Psc on timescales of up to 3000 days. There are minima at multiples of 550 days, indicating a coherent LSP of this length. 
RZ Ari.-Percy et al. (2003) obtained periods of 37.7 and 56.5 days, from analysis of individual seasons of APT photometry as well as from the entire APT data set. These periods are clearly visible in the power spectrum of the merged data (Fig. 2). The LSP is not well defined (the amplitude is only 0.02), but appears to be $370 \pm 10$ days. This is suspiciously close to a year, but is probably real, as there are no obvious instrumental effects that would produce a one-year period in photoelectric data. Percy et al. (1996) reported a LSP of "about 500 days."

$\eta$ Gem.- The pulsation period is 234 days; the power spectrum shows this, and much smaller alias peaks. The SCD is also relatively simple, showing regular minima at multiples of 234 days. There is no evidence for additional pulsation periods or for a LSP.

V614 Mon.- The power spectrum and SCD are complex; the primary pulsation period is $90 \pm 1$ days, as others have found, but it appears that there is an additional pulsation period, perhaps $67 \pm 1$ days. There is no convincing LSP; on timescales from 500 to 5000 days, the SCD is absolutely flat (Fig. 3).

$R S$ Cnc.- The power spectrum and SCD both point toward a primary period of 225 days, but both are complex; there is a possible second pulsation period of 137 days. The SCD from 500 to 5000 days shows no obvious long secondary period.

VY UMa.-In the power spectrum, there are peaks at 125 and 188 days, as PWH reported. These differ in frequency by one cycle per year; the SCD shows that the 125-day period is the dominant one, but the complexity of the SCD suggests that there may actually be a second pulsation period at or near 188 days. On timescales of 500 to 5000 days, there are no coherent minima in the SCD greater than 0.01 mag.

FS Com.-Percy et al. (2003) reported periods of 38.2 and 55.4 days from analysis of individual seasons of APT photometry, and of the entire APT data set, and these periods are clearly present in our power spectrum. There is also a very well-defined LSP in the SCD, and the power spectrum (Fig. 4), at $680 \pm 10$ days. PWH reported a long period of 600-750 days.

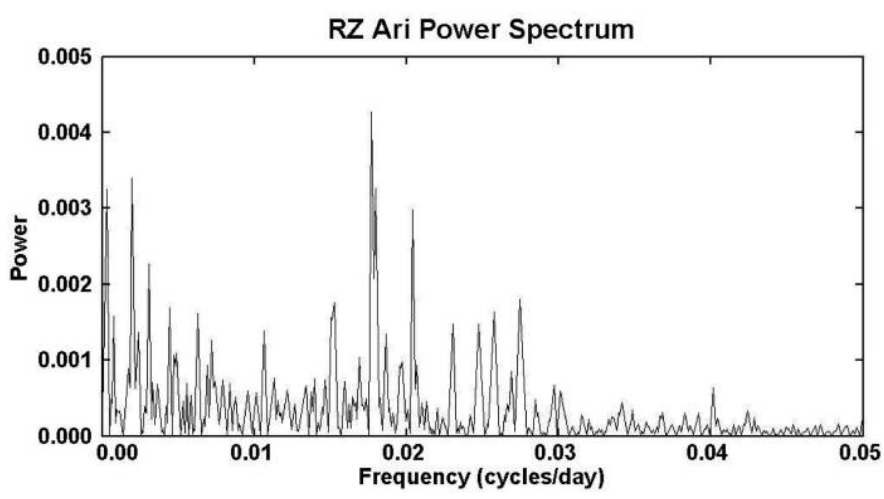

FIG. 2.-Power spectrum of RZ Ari, showing periods of 56.5 days ( $0.0177 \mathrm{cy}$ cles per day), 37.7 days ( 0.0265 cycles per day), and 370 days ( 0.00270 cycles per day).

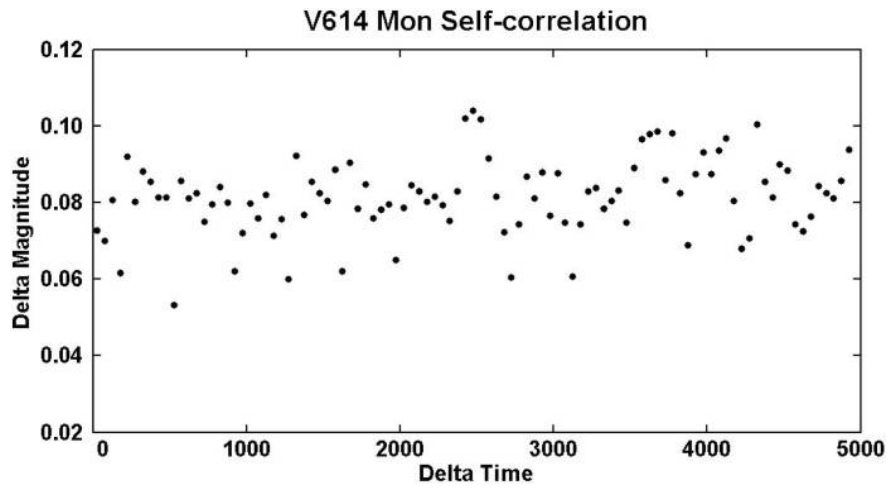

FIG. 3.-SCD of V614 Mon on timescales of up to 5000 days. There are no repeating minima greater than about $0.01 \mathrm{mag}$, indicating no coherent LSP.

SW Vir.-There is only one period, 154 days, in both the power spectrum and the SCD.

$R$ Lyr.-There are two peaks in the power spectrum, at 64.3 and 46 days, the most prominent being the longer one. The measurements are dense and numerous, so aliasing is minimal, and so it appears that both periods are real. Their ratio is consistent with first and second overtone pulsation. PWH reported "46 and/or 64 days." On timescales of hundreds to thousands of days, the SCD is flat to \pm 0.01 ; there is no evidence for a coherent LSP.

EU Del.-There is only one period, 61.6 days, which was also found by Percy et al. (1996) and PWH. $O-C$ analysis suggests that the period is actually 62.4 days. There is no evidence for additional pulsation periods, or for a LSP. The light curve does, however, vary from cycle to cycle; the cause is not known.

V1070 Cyg.-Both the power spectrum and the SCD are complex, and suggest the presence of two or more lowamplitude pulsation periods. The dominant one seems to be about 64 days, with the possibility of one longer and one shorter

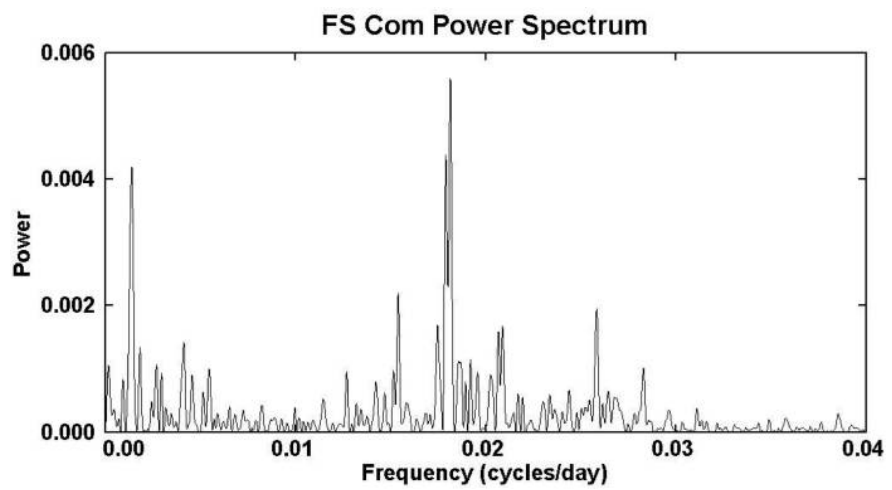

FIG. 4.-Power spectrum of FS Com, showing periods of 38.2 days (0.0262 cycle per day), 55.4 days ( 0.0181 cycle per day), and 680 days (0.00147 cycle per day). 
pulsation period — perhaps 100 and 42 days. PWH reported " 60 , $50 \pm$, complex." On timescales of 200 to 2000 days, there is no evidence in the SCD for a coherent LSP.

$W$ Cyg.-There is one dominant period, 131 days, in the power spectrum, but there are several lower-amplitude peaks at longer periods. As noted by PWH and references therein, the star shows periods of about $125 \pm 5$ and $250 \pm 10$ days and may slowly switch between these two modes, as does R Dor with periods of 175 and 332 days (Bedding et al. 1998). Indeed, our SCD shows alternating deep and shallow minima on a timescale of 130 days - something that might be expected if these two periods were present-and one of three smaller peaks in the power spectrum is at a period of 250 days. There is no LSP, though we note that the amplitude of the 131-day variation rises and falls on a 5000-day timescale, being smallest at JD 2446000 and JD 2451000 and largest at JD 2449000 and JD 2454000.

W Cyg is typical of most of the stars in our sample, in the sense that it shows very slow and small variations in the amplitude and mean magnitude of the light curve on timescales of

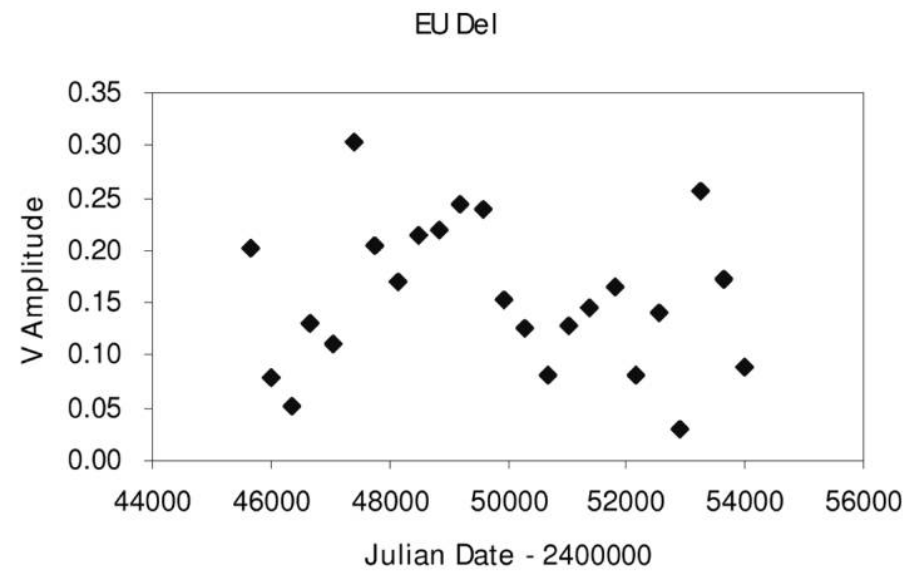

RZ Ari (37.7 day period)

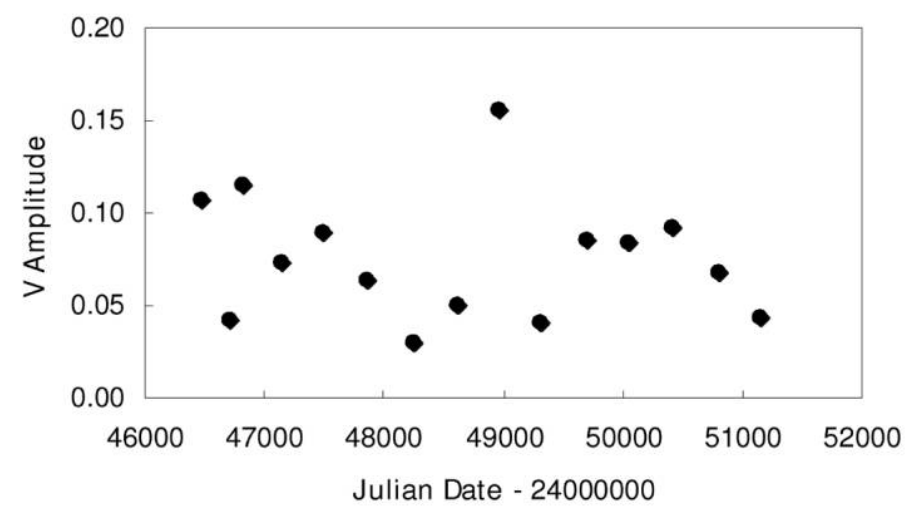

typically 3000-5000 days. Other stars showing such variations are: RZ Ari, V614 Mon, RS Cnc, VY UMa, FS Com, SW Vir, R Lyr, EU Del, and V1070 Cyg.

\subsection{Amplitude Variability}

In an earlier study, Percy et al. (2003) investigated the amplitude variability in a small number of small-amplitude pulsating red giants with two or three periods. They found that the amplitudes changed significantly on timescales of 2000-3500 days. Most often, the amplitudes of multiple modes in a single star rise and fall in unison.

We have reinvestigated this phenomenon in a few stars, using our longer and denser data sets. We use the least-squares fitting routine in the Period04 package (Lenz \& Breger 2005).

Figure 5 shows the time variability of the amplitudes in three stars, including the two different periods in RZ Ari. As found earlier, there is long-term variability on a timescale of seasons (years); this can be seen from the light curves. In the case of EU Del, we tried subdividing the data from each season, and

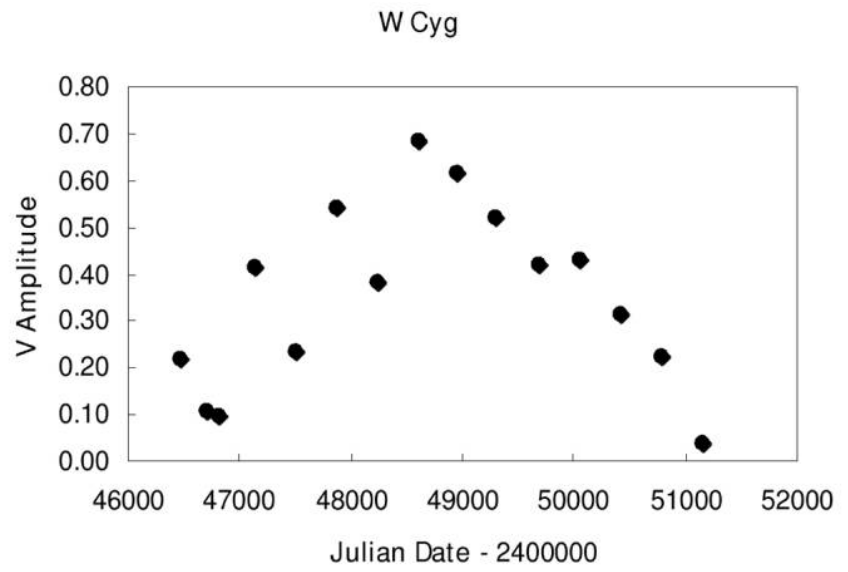

RZ Ari (56.5 day period)

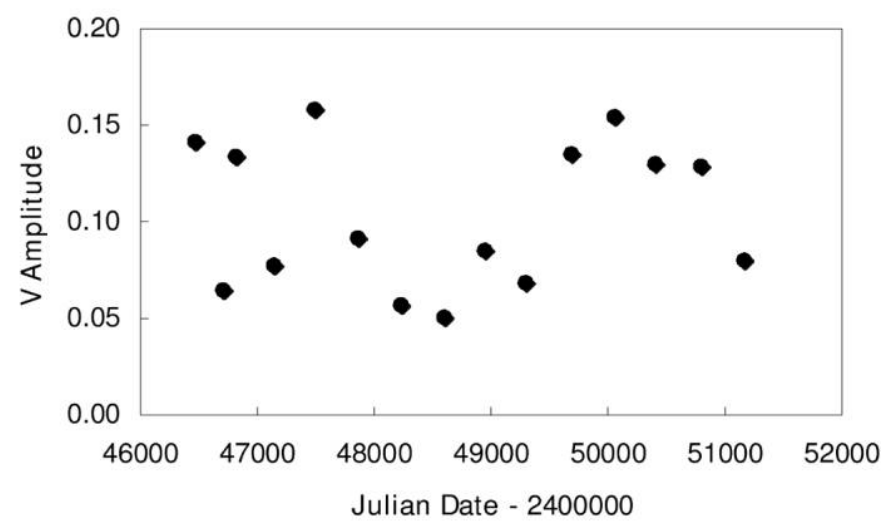

FIG. 5.-Pulsational amplitude variability, with time, in three red giants, EU Del, W Cyg, and the two dominant periods in RZ Ari. There are variations on a timescale of years, but also possible variations on a timescale of half a season. See the electronic edition of the PASP for a color version of this figure. 
looking for variability on a timescale of less than a season. It is clear, from the light curve, that there are cycle-to-cycle changes in amplitude, even though the star is monoperiodic. In all three stars shown in Figure 5, there appear to be smaller short-term changes, in addition to the long-term ones.

Inspection of the light curve of W Cyg shows that the amplitude reached a minimum at about the time that the APT data ended but, since then, has started to increase, the period remaining at about 130 days. The variability has, however, become less regular. The AAVSO visual measurements are useful for monitoring the behavior of the star.

\subsection{Wavelet Analysis}

Percy \& Kastrukoff (2001) previously used wavelet analysis to study possible mode-switching and amplitude variability in a few small-amplitude pulsating red giants. Using the AAVSO photoelectric photometry alone, the results were limited by seasonal gaps that can extend for two or more cycles. The most significant period was usually the well-known 62.5-day one, but it occasionally switched to a $77 \pm 2$ day alias, which we believe was an artifact of the star's irregularity, small amplitude, and seasonal gaps. We repeated the analysis using 5-day means of AAVSO visual data, for which the seasonal gaps were less severe. The most significant period was almost always the 62.5-day one, but 77-day and 95-day aliases occasionally appeared.

We have used our longer, denser data sets to reinvestigate a few of these stars. We have used the wavelet software available on the AAVSO Web site (Foster 1996). EU Del provides a useful starting point, because it is monoperiodic, and its data set is long and dense (though with seasonal gaps). Simple inspection of the light curve shows that the amplitude rises and falls on a timescale of 7-8 years. Figure 6 shows the wavelet plot; it confirms that the amplitude of the 62.5-day period rises and falls between JD 2447000 and 2451000, and that there are no other periods, except alias periods.

For the remaining stars, the wavelet plots are very complicated, and clearly affected by the large seasonal gaps; they provide no useful information. We conclude that wavelet analysis is not an effective tool for analyzing this type of star, with this kind of data.

\subsection{Phase Diagrams}

Partly as an afterthought, we determined phase diagrams for a few of the monoperiodic stars, to investigate the nature of the irregularity. For EU Del, the phase diagram simply showed scatter around the mean curve for the 62.45-day period. For $\eta$ Gem, however, the majority of the points lay on a smooth curve, and a small fraction of the points lay below, detached from the mean curve. This suggests that, for this star, there are a few epochs when this star is significantly fainter than usual. The three most prominent are: JD 2443170, 2447270, and 2451240. We note that $\eta$ Gem is a binary with a period of 2983 days (McLaughlin \& Van Dijke 1944), though the fadings do not appear to occur with this period and are separated by about 4000 days.

\subsection{Period Fluctuations in EU Del}

The period changes of large-amplitude Mira stars have been studied in detail, using the long database of visual observations by the AAVSO. The $O-C$ diagrams are dominated by random cycle-to-cycle period fluctuations (Eddington \& Plakidis 1929; Percy \& Colivas 1999a, 1999b and references therein). It is not known whether similar fluctuations are found in smallamplitude pulsating red giants.

Because the data set for EU Del was longest and densest, we measured as many times of maximum brightness as possible by visual inspection of the light curve. We first plotted an $O-C$ diagram, using a period of 62.4 days. The diagram was horizontal, indicating that the 62.4-day period was valid. It also showed no obvious curvature, indicating no measurable long-term period change. There were random excursions of \pm 10 days. In order to determine whether these were due to observational errors in determining the times of maximum or due to random cycle-to-cycle period fluctuations, we applied the EddingtonPlakidis algorithm to the data, using a program written in Excel $^{\mathrm{TM}}$ by Deepak Chandan. The result is shown in Figure 6.

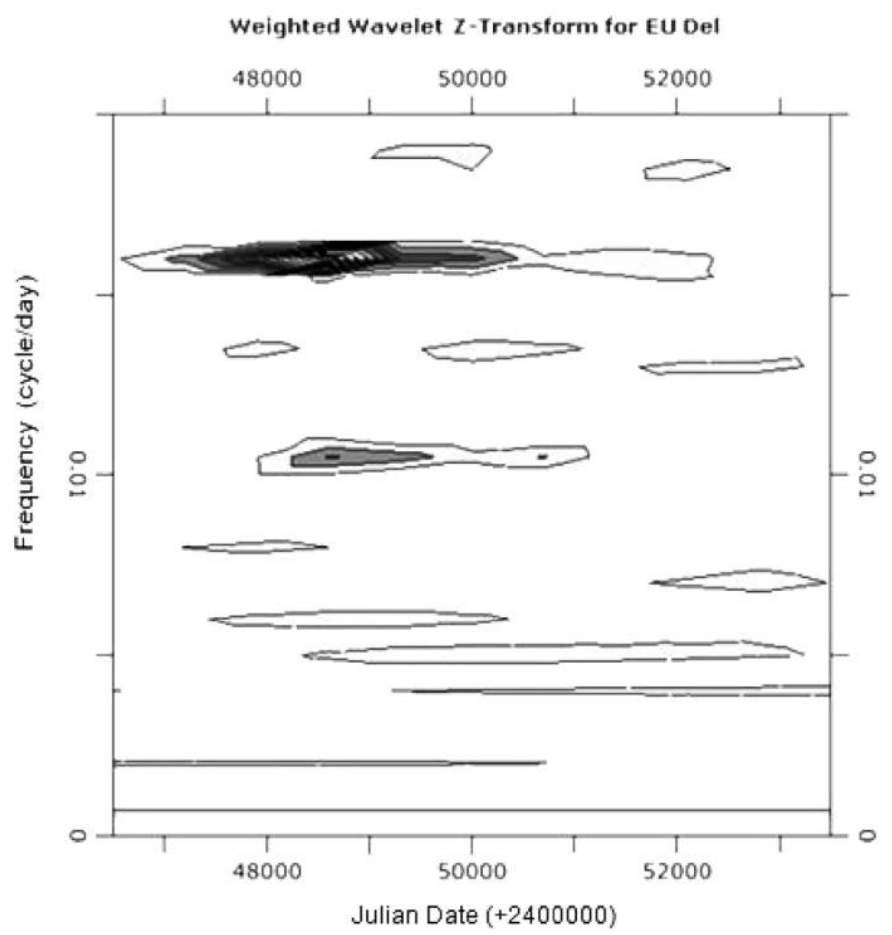

FIG. 6.-Wavelet plot for EU Del. The amplitude of the 62.5-day period ( 0.016 cycle per day) rises and falls. There are no other periods, other than the aliases, that are a result of the seasonal gaps in the data. See the electronic edition of the PASP for a color version of this figure. 
If random cycle-to-cycle period fluctuations were dominant, then the graph would be a straight line with positive slope. The graph is clearly not a straight line. The nominal slope of Figure 6 is actually not significantly different from zero. This indicates that there are no significant period fluctuations. The $3 \sigma$ upper limit to the average fluctuation per period is 2.73 days, or 0.04 cycle. Most Mira stars have fluctuations of 0.04 or less (Percy \& Colivas 1999a, 1999b). Figure 6, however, does not support the hypothesis that EU Del undergoes random cycle-tocycle period fluctuations.

\subsection{Color Variations}

PWH previously carried out a period analysis on the APT $V$ photometry. Here, we determine the relative amplitudes in $V, R$, and $I$ of pulsation periods in 16 stars, and long secondary periods in 12 stars. We are especially interested in whether the LSPs show the same color behavior as the short radial pulsation periods. The average amplitudes and their standard errors were determined using the least-squares fitting routine in Period04.

Figures 7 (short periods) and 8 (long periods) show a selection of results. In each case, the amplitude behavior is similar; the amplitude decreases from $V$ to $R$ to $I$. Within the error bars, the slope of the decrease is the same; specifically $\Delta V \sim 1.8 \pm$
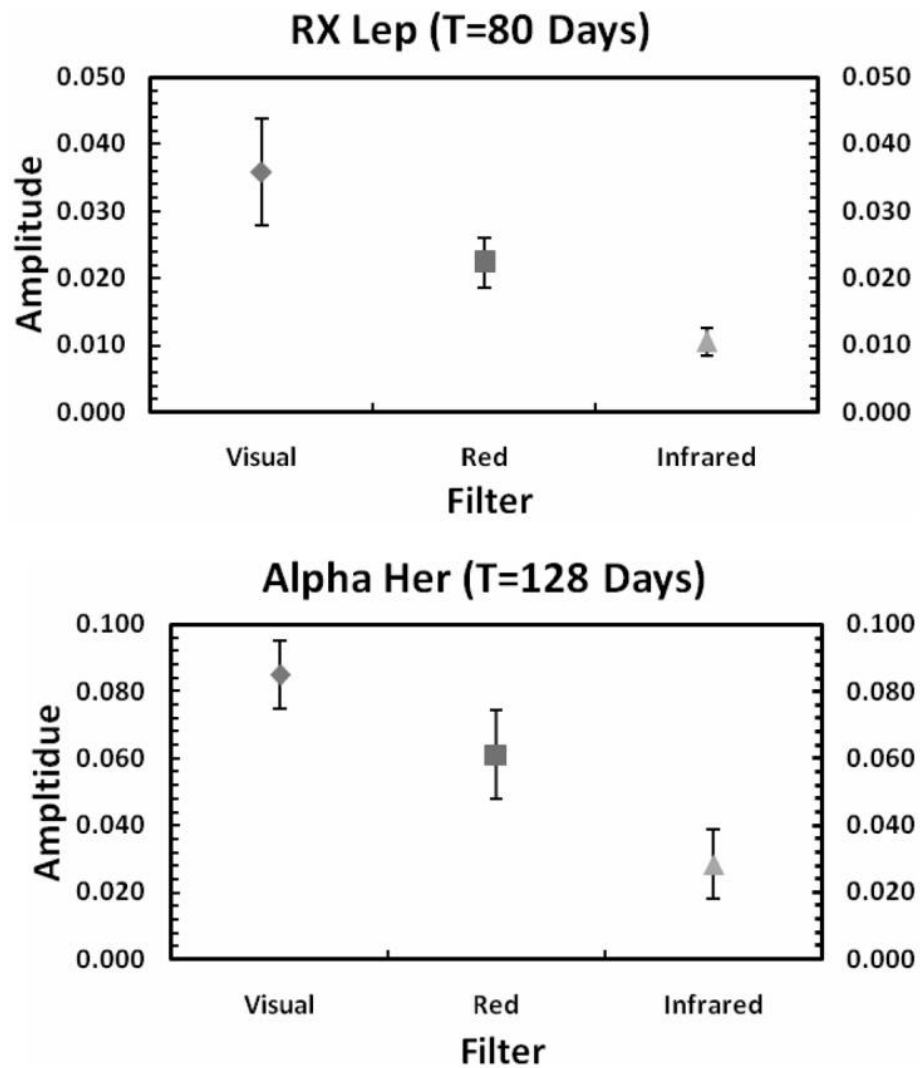

$0.2 \Delta R$ and $\Delta R \sim 1.8 \pm 0.2 \Delta I$. This is true for both the short periods and the long periods. The only possible exception to this result is the 242-day period of EG And, which appears to be connected with binary motion. For this star, the decrease is marginal at best (Figure 8).

\subsection{Additional Period Information}

In the course of analyzing the $R I$ data, we obtained some information which improved or extended the period determinations by PWH: $\alpha$ Her: the LSP may be about 1500 days. $B C$ CMi: the LSP appears to be about 250 days. $\mu$ Gem: there is no evidence for an LSP. UX Lyn: the LSP may be about 1200 days. Y Lyn: the LSP appears to be closer to 1300 days than to the 1400-day period given by PWH. 30 Her: the periods may be 87 and 880 days, rather than 93 and 833 days as given by PWH. $\psi$ Aur: the periods are 300 and 2400 days. FS Com: the LSP may be 725 rather than 680 days given by PWH. V642 Her: the LSP may be 1500 days. $T U C V n$ : the LSP is 350-400 days.

\section{DISCUSSION}

Some of the stars in our sample are superficially simple. For instance, EU Del shows no evidence for secondary periods,
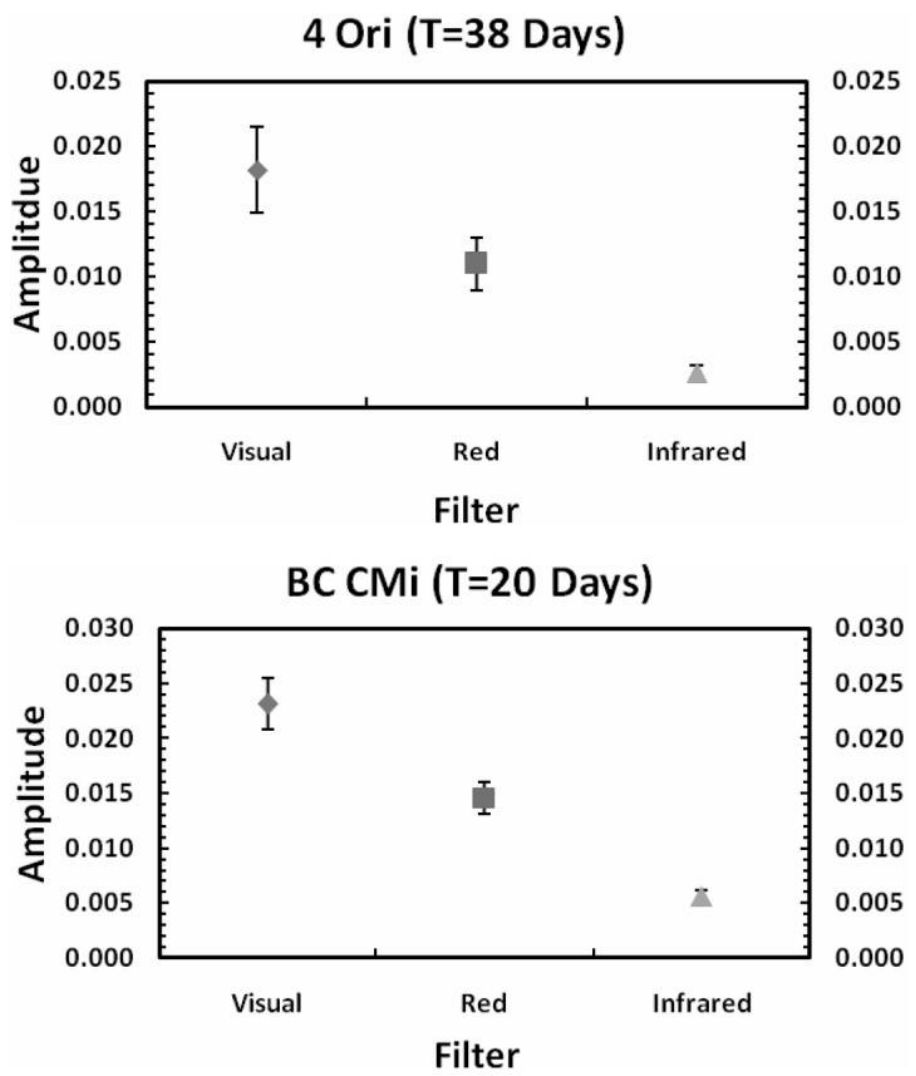

FIG. 7.-Amplitude of the pulsational variations of the red giants RX Lep, 4 Ori, $\alpha$ Her, and BC CMi as a function of wavelength (filter): $V$-yellow, $R$-red, and $I$-near-infrared. See the electronic edition of the PASP for a color version of this figure. 

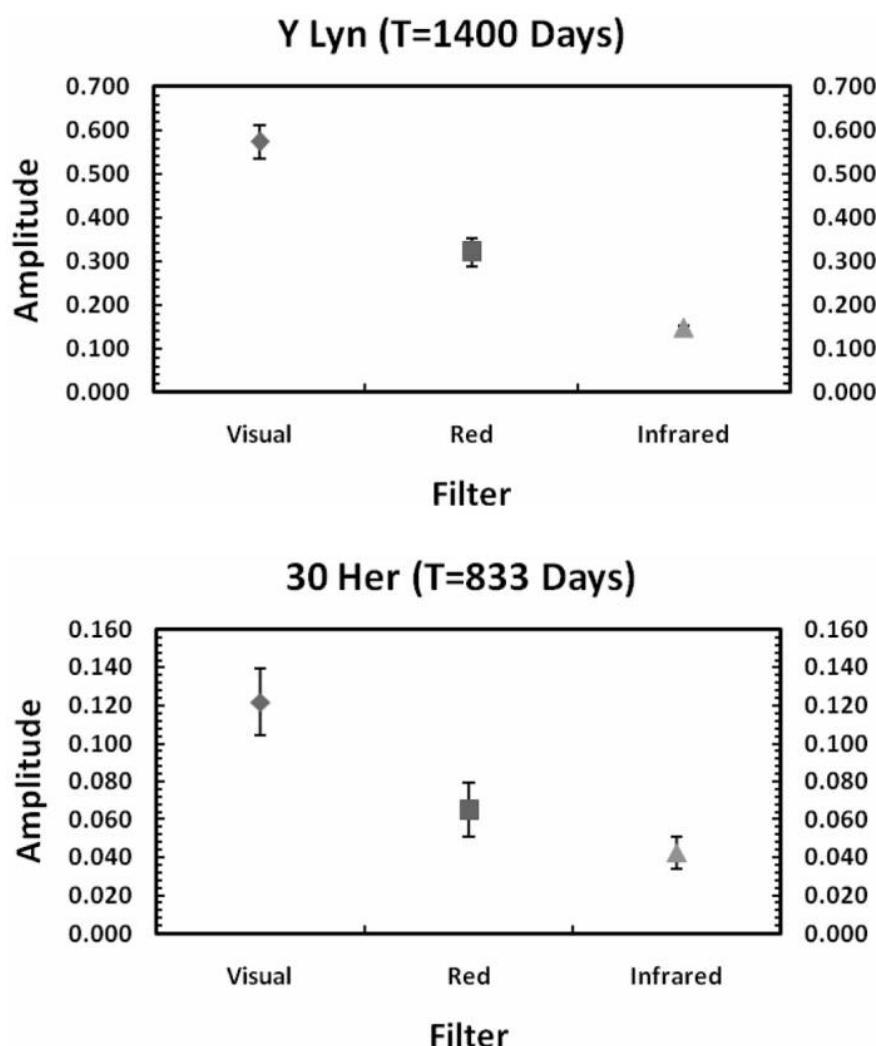
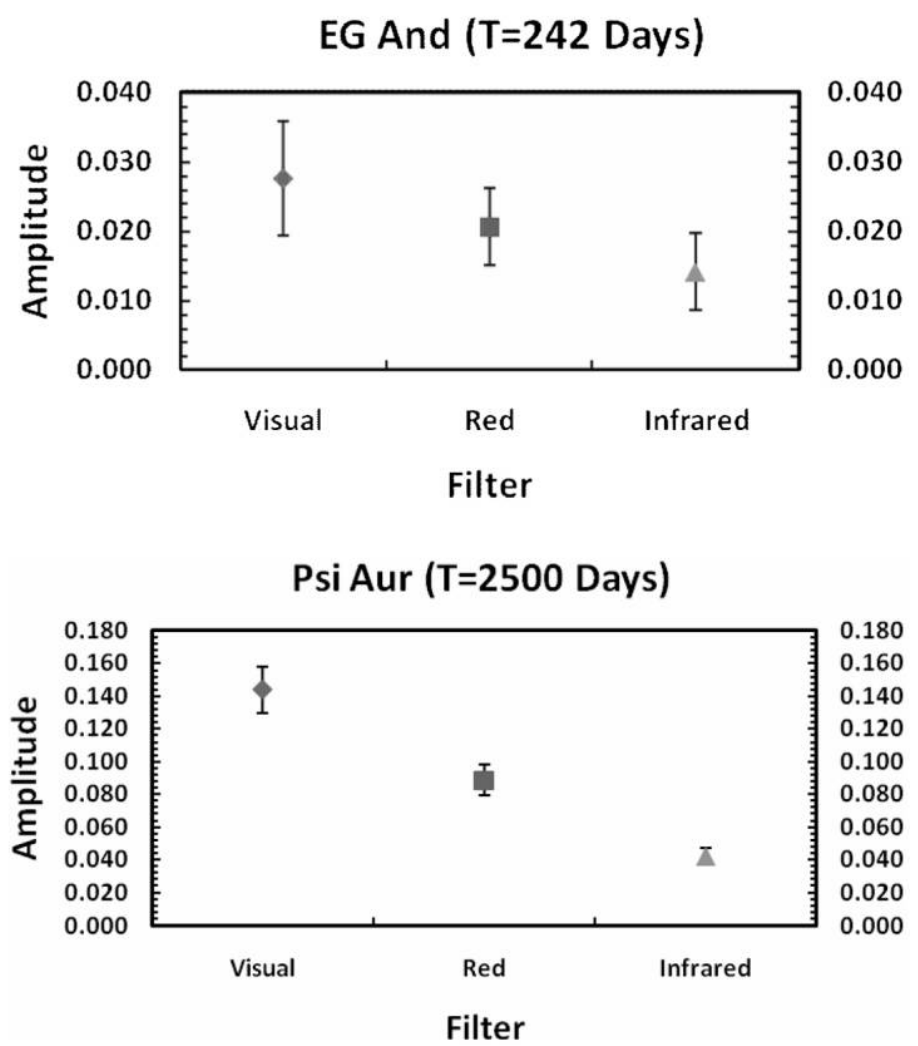

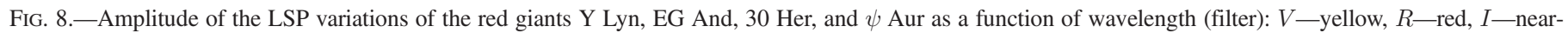
infrared. See the electronic edition of the PASP for a color version of this figure.

either short or long. Yet the light curve varies from cycle to cycle; the cause is not known. The period, however, is constant. There are no detectable secular period variations, or random cycle-to-cycle fluctuations. The scatter in the $O-C$ diagram can be explained by the observational errors in determining the times of maximum.

We investigated the long-term amplitude variability in several stars, using least-squares and wavelet analysis. Unfortunately, the long seasonal gaps present problems for both techniques. Although the dominant timescales of variability are 2000-3500 days, there do appear to be cycle-to-cycle changes as well. In some stars, these are due to multiperiodicity. We can separate the individual periods in a few stars, and show that their amplitudes vary on long timescales. EU Del is not multiperiodic; its cycle-to-cycle changes must have some other cause.

Four of the 13 stars show LSPs; the rest do not show coherent variability of this kind. This is consistent with other studies that show LSPs in about a third of PRGs.

The cause of the LSPs remains unknown, though, in the case of EG And, it is clearly related (in some way) to binarity. Other possible causes listed (and rejected) by Wood et al. (2004) are: radial pulsation, nonradial pulsation, various consequences of binarity, rotation of a prolate spheroid, episodic dust ejection, and starspot cycles. Or perhaps there is some unknown mechanism, connected with the complex physical processes in the envelopes of these stars.

Very slow (thousands of days), small variations in amplitude and mean magnitude are another characteristic of most of these stars. These are so small and so slow that they could arise from a wide range of possible causes. Episodic dust ejection, or the rotation of a star with a nonuniform photosphere are possibilities. Because their timescales are so long, it is not possible to know whether the variations are periodic or not. Further decades of observations would be useful. In particular, they might help to determine whether there is any correlation between the very long-term changes in amplitude and mean magnitude; the existing data sets are not quite adequate for this purpose.

In the 16 stars that we have analyzed, the pulsation amplitude decreases with decreasing wavelength, from $V$ to $R$ to $I$. We expect this behavior, because we are observing these stars on the blue side of their spectral energy distribution. The same is true of Mira stars, except that the relative $V$ amplitude is even larger, because of the temperature sensitivity of the TiO bands in the spectra. The LSPs-whatever their cause-show the same behavior, except in the case of EG And, known to be a binary. 


\section{CONCLUSIONS}

We have shown that merged photometric data sets, typically $8000+$ days long, can yield improved information about the presence and nature of long secondary periods, and multiple pulsation periods in PRGs. In some cases, even such long data sets cannot "solve" stars with multiple pulsation periods and/or noncoherent LSPs. Most of the stars have variations on even longer timescales-thousands of days. Such variations are only apparent in data sets like ours. The amplitudes of both the pulsation periods and the LSPs show the same color behavior: the amplitude decreases with increasing wavelength.
This work would not have been possible without the contributions of dozens of AAVSO photoelectric photometry observers, and of the AAVSO Headquarters staff who reprocessed the data and made them available to us. We acknowledge a research grant from the Natural Sciences and Engineering Research Council of Canada, and the support of the Ontario Work-Study Program. Coauthors M. M., C. O. N., R. P., and R. S. were undergraduate research students at the University of Toronto. We thank Deepak Chandan for making his results available to us. This research has made use of the SIMBAD database, operated at CDS, Strasbourg, France.

\section{REFERENCES}

Bedding, T. R., Zijlstra, A. A., Jones, A., \& Foster, G. 1998, MNRAS, 301, 1073

Eddinton, A. D., \& Plakidis, S. 1929, MNRAS, 90, 65

Fekel, F. C., Joyce, R. R., Hinkle, K. H., \& Skrutskie, M. F. 2000, AJ, 119,1375

Foster, G. 1996, AJ, 112, 1709

Hoffleit, D., \& Jaschek, C. 1991, The Bright Star Catalog (New Haven: Yale Univ. Observatory)

Kiss, L. L., Szatmary, K., Cadmus, R. R.Jr., \& Mattei, J. A. 1999, A\&A, 346, 542

Kurtz, D. W., \& Pollard, K. R. 2004, ASP Conf. Ser. 310, Variable Stars in the Local Group (San Francisco: ASP), 588

Lenz, P., \& Breger, M. 2005, Commun. Asteroseismology, 146, 53

McLaughlin, D. B., Van Dijke, \& S. A. E. 1944, ApJ, 100, 63

Percy, J. R., Bakos, A. G., Besla, G., Hou, D., Velocci, V., \& Henry, G. W. 2004, in ASP Conference Series 310, Variable Stars in the Local Group, ed. Kurtz, D. W., \& Pollard, K. R. (San Francisco: ASP), 348
Percy, J. R., Besla, G., Velocci, V., \& Henry, G. W. 2003, PASP, 115,479

Percy, J. R., \& Colivas, T. 1999a, PASP, 111, 94

1999b, IAU Inf. Bull. Var. Stars, 5041

Percy, J. R., Desjardins, A., Yu, L., \& Landis, H. J. 1996, PASP, 108, 139

Percy, J. R., Dunlop, H., Kassim, L., \& Thompson, R. R. 2001a, IAU Inf. Bull. Var. Starsm 5041, 1

Percy, J. R., \& Kastrukoff, R. 2001c, JAAVSO, 30, 16

Percy, J. R., Wilson, J. B., \& Henry, G. W. 2001b, PASP, 113, 983 (PWH)

Waagen, E. O. 2006, AAVSO Photoelectric Photometry Newsletter, 24, No. 1, http://www.aavso.org/observing/programs/pep/ pepnewsletter/jul06.shtml\#aavso

Wood, P. R., Olivier, A.E., \& Kawaler, S. D. 2004, ApJ, 604, 800 